\title{
Endovascular Management of a Rare Cause of Pelvic Hemorrhage
}

\author{
Agamya Saxena ${ }^{1}$, Manish Jain ${ }^{2}$ \\ ${ }^{1}$ Department of Interventional Radiology, Siddhanta Red Cross Superspeciality Hospital, Bhopal, Madhya Pradesh; ${ }^{2}$ Department of \\ Gastromedicine, Asian Globus Hospital, Bhopal, Madhya Pradesh, India.
}

\section{Corresponding Author:}

Dr. Agamya Saxena

Email: agamya.saxena@gmail.com

This is an Open Access article distributed under the terms of the Creative Commons Attribution License (creativecommons.org/ licenses/by/3.0).

Received Accepted

Published

April 17, 2019

July 17,2019

July 25, 2019

\begin{abstract}
Background: Pelvic hemorrhage has a plethora of causes. Although an iatrogenic cause is not uncommon, most cases refer to a post-partum state as the common scenario. A post-hysterectomy pseudoaneurysm of uterine artery has been very rarely reported. The delayed presentation of hemorrhage can confuse the picture all the more. Timely angiographic detection and endovascular treatment of such patients can save them from a more invasive open surgery. Case Report: A young female presented with hemodynamic shock and fall in hemoglobin with a history of radical hysterectomy about one month back. Despite a normal appearing CT angiogram, she was taken up for conventional angiography as the index of suspicion of vascular leak was high. On pelvic angiography, she was diagnosed with a right uterine artery pseudoaneurysm, which was embolized with coils in the same sitting. Later, she got discharged from hospital, without having undergone any further intervention. Conclusion: Angiography proved an invaluable tool, not just to determine the cause of bleeding in this case, but also to embolize the bleeding artery. This rare case scenario should be kept in mind in post-hysterectomy patients who present with significant hemorrhage even in delayed settings.
\end{abstract}

Keywords: Hemorrhage, Hysterectomy, Interventional Radiology, Aneurysm, Uterine Artery Embolization.

\section{Introduction}

Hysterectomy is a widely performed procedure across the world, with a definitive list of associated complications. Among these, a pseudoaneurysm of uterine artery is one of the rarest complication. The case report describes a patient with large pelvic hematoma, which heralded with hemodynamic shock and a rapid drop in hemoglobin. Surgical evacuation of this hematoma would have had the risk of "loss of tamponade" and further uncontrolled bleed. Despite a normal computerized tomography [CT] angiogram, a prompt decision to radiologically "explore" the pelvic vasculature was taken. Digital substraction angiography [DSA] proved to an invaluable tool to uncover a pseudoaneurysm of the right uterine artery. Uterine artery embolization is otherwise, an established procedure for fibroids and post-partum hemorrhage. In this case, embolization with PVA particles and coils was performed via bilateral uterine arteries for the pseudoaneurysm, through a single right femoral artery puncture. The case underlines the importance of interventional radiological embolization of life-threatening pelvic hemorrhage, such that the patient does not have to undergo any major surgery.

\section{Case Report}

A 32 year old female presented with two days history of acute abdominal pain. The pain was localized in right iliac fossa and pelvic region. On examination, moderate abdominal tenderness was also present, along with abdominal distention. She had a history of hysterectomy performed about one month ago for symptomatic uterine fibroids. An urgent ultrasonography of abdomen was performed 
which revealed gross amount of echogenic fluid in pelvic cavity. Thick mobile echoes suggested a possibility of hemoperitoneum.

The diagnosis was supported by initial hemodynamic shock and anemia. The BP at presentation was $90 / 60 \mathrm{~mm} \mathrm{Hg}$, with tachycardia [heart rate: $120 \mathrm{bpm}$ ], and a hemoglobin $(\mathrm{Hb})$ value of $8.3 \mathrm{gm} / \mathrm{dL}$. Her previously recorded $\mathrm{Hb}$ was 12.7 $\mathrm{gm} / \mathrm{dL}$ which suggested a rapid onset bleeding. After initial resuscitation with intravenous fluids and blood transfusion, she got stabilized. She was further investigated with CECT abdomen with angiography protocol. The CT scan confirmed a pelvic collection causing compression over right ureter and causing right moderate hydronephrosis [Fig.1]. Her right iliac fossa and flank pain could be explained by this finding. However, the CT angiogram images did not reveal any significant ooze, active leak or pseudoaneursym arising from any pelvic artery [Fig.2].

A decision to surgically evacuate this hematoma was not appreciable due to the risk of loss of the "tamponade" effect of hematoma over bleeding vessel. Hence, it was decided to take the patient for conventional catheter-based digital substraction angiography [DSA], and look for any culprit artery. Two days after the initial presentation, the patient underwent DSA. A right femoral artery access was taken with a $6 \mathrm{~F}$ sheath. Using 0.035 " angled-tip glidewire [Terumo] and $5 \mathrm{~F}$ pigtail catheter, aortogram was performed for a general pelvic vasculature assessment, and also to opacify the ovarian arteries [Fig.3]. Further, catheterization of each of the uterine arteries was done with a $5 \mathrm{~F}$ Robert's uterine catheter [RUC] and hydrophilic guide wire. Selective angiograms of both uterine arteries were performed which revealed diffusely increased vascular "blush", tortuosity of arterial branches and overall hypervascularity [Fig.4]. A 2.7 French microcatheter and microguidwire combination [Progreat, Terumo] was used to superselectively catheterize each uterine artery. The tip of catheter was placed distal to the origin

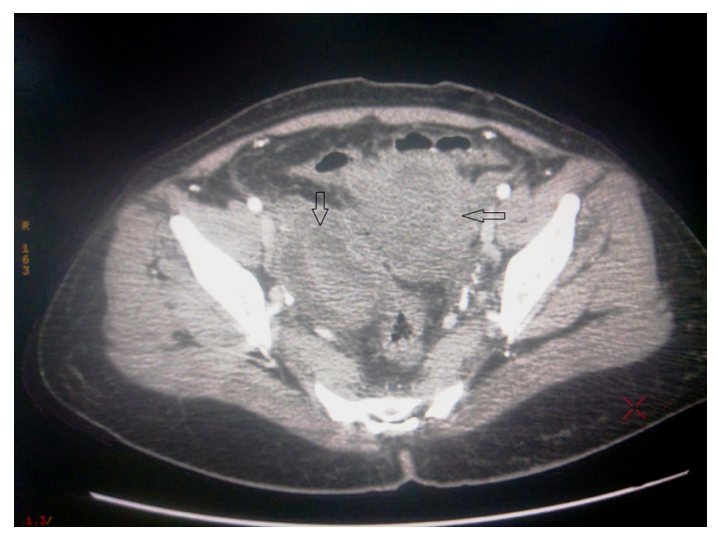

Fig.1: CECT pelvis showing multi-layered densities depicting hemorrhage [arrows].

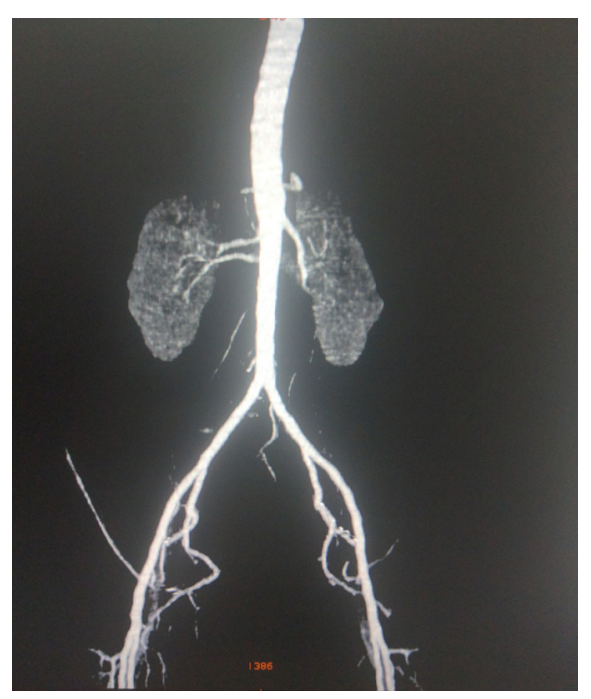

Fig.2: Coronal volume rendered image of CT angiography showing no gross abnormality.

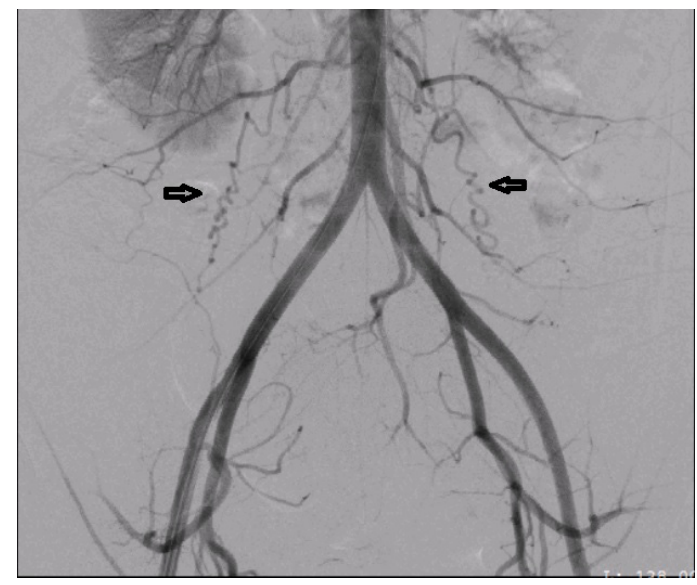

Fig.3: DSA aortogram and pelvic angiogram showing normal ovarian arteries on both sides [arrows]. 
of cervico-vaginal artery, to prevent inadvertent vaginal ischemia and future possibility of vaginal parasthesia. 500-700 micron sized polyvinyl alcohol [PVA] particles were used to embolize this hypervascularity from both sides. The PVA was injected with gentle, repeated push of the syringe, and continued till stasis in the main artery was achieved.

To our surprise, a post-embolization check angiogram revealed opacification of a pseudoaneurym arising from main trunk of right uterine artery [Fig.5]. Hence, a decision of coil embolization was taken. A $5 \mathrm{~cm}$ long, $5 \mathrm{~mm}$ diameter coil [35-5-5; MReye, Cook] was used to coil the right uterine artery [Fig.6]. As there was possibility of retrograde filling of the pseudoaneurysm from

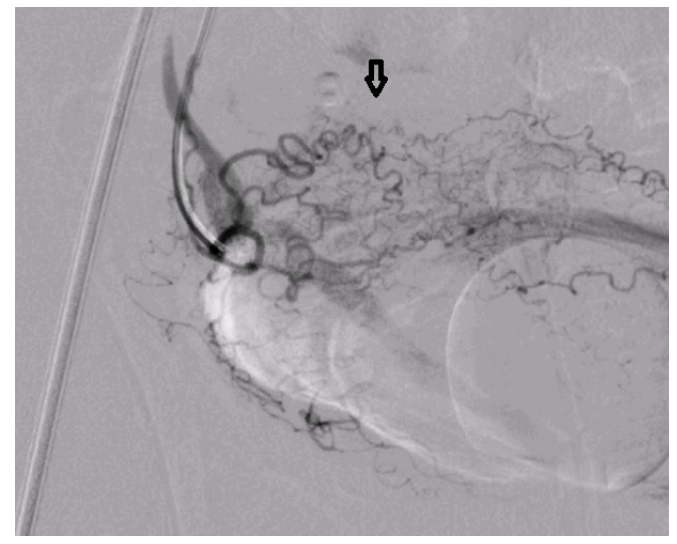

Fig.4: Super-selective right uterine artery DSA showing tortuous branches, with hypervascularity [arrow].

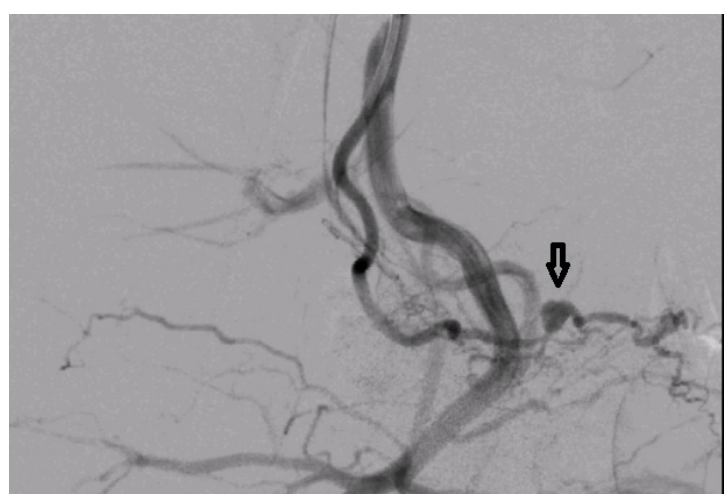

Fig.5: DSA of right uterine artery showing the irregular shaped pseudoaneurysm seen as focal "dilatation" [arrow]. the opposite side, the anterior division of left internal iliac artery was also embolized using $7 \mathrm{~cm}$ long, 6 mm diameter coil [35-7-6; MReye, Cook]. Post-embolization final angiogram revealed nonopacification and exclusion of pseudoaneurysm from circulation [Fig.7].

The patient was then shifted to ICU for next two days, and then shifted to ward. Owing to rapid decrease in abdominal distention and pain, and a stable hematocrit, she was discharged from hospital five days after the embolization procedure.

\section{Discussion}

In contrast to true aneurysms, pseudoaneurysms are not surrounded by the normal three vascular wall layers of tunica intima, media, and adventitia.

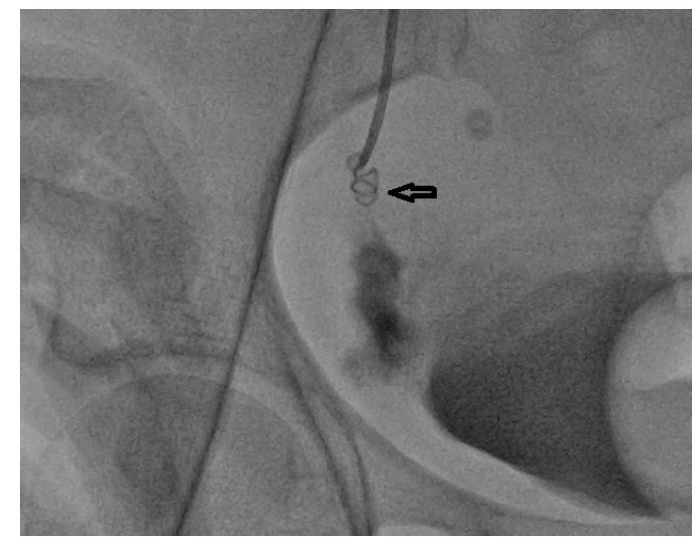

Fig.6: A stainless steel coil being deployed in right uterine artery [arrow].

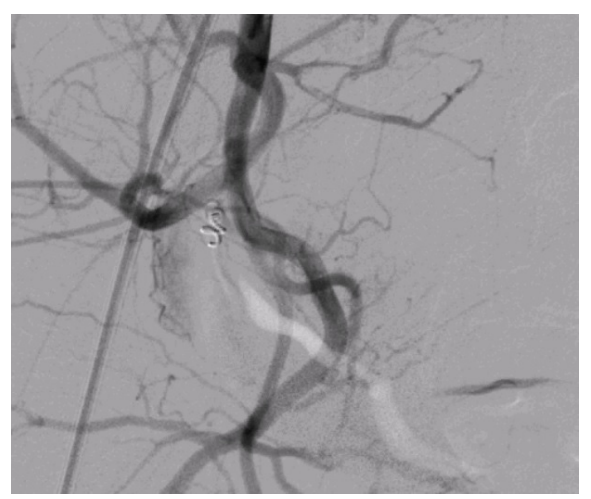

Fig.7: Post-coiling internal iliac angiogram confirming exclusion of pseudoaneursym [arrow]. 
They are bordered by adjacent perivascular tissue and thrombus, creating a pseudocapsule. They can undergo spontaneous thrombosis and resolution, or become infected and develop into a septic pelvic collection. Alternatively, they can cause pain and pressure symptoms to nearby organs, like in our case where the hematoma compressed over right ureter. Occasionally, they can be asymptomatic and present as an incidental finding on routine post-operative imaging [1]. Spontaneous rupture is the most serious complication with high morbidity and mortality. Depending on the cause of the pseudoaneurysm and the type of the antecedent operation, rupture presents with intra- or extraperitoneal bleeding, uncontrolled metrorrhagia or vaginal vault bleeding. Pelvic surgery is the most common cause of uterine artery pseudoaneurysms. In obstetrics, they can present with secondary postpartum hemorrhage after caesarean section $[2,3]$; in gynaecology, they can develop posthysterectomy, myomectomy or dilatation and curettage [4-9].

The natural history involves injury to a vessel during dissection or suturing, incomplete vascular sealing or extensive desiccation which results in the development of hematoma. Central liquefaction of the hematoma leaves a cavity which communicates with the parent vessel [1]. Rupture is unpredictable and characteristically delayed, as time is needed for this process to take place and the pseudoaneurysm to form. Diagnosis is suspected from the clinical history and confirmed with imaging investigations. Ultrasound scan is usually the first line investigation. Magnetic resonance imaging and angiography (MRA) can be used to confirm the diagnosis in cases where CTA cannot be done. Digital substaction angiography is the gold standard diagnostic technique [10]. Color Doppler ultrasound shows color signals within the lesion and aliasing at the neck of pseudoaneurysm due to turbulent blood flow. Spectral Doppler reveals a characteristic to-and-fro pattern, and it has been reported to have a diagnostic sensitivity of 95\% [11-
13]. If pseudoaneurysm is not connected with the endometrial cavity, hemorrhage may be confined to the abdominal cavity, leading to abdominal pain, as in our case [11].

Treatment of uterine artery pseudoaneurysm is done by uterine artery embolization or ligation, internal artery ligation or by emergency hysterectomy in cases of life-threatening vaginal bleeding and/or wherever family of the patient is completed. Uterine artery embolization is the treatment of choice with very good success rate in occluding the affected vessel. Surgery should be avoided as it can delay definite treatment and result in further bleeding and inadvertent ureteral and/or bladder injury caused by the application of multiple stitches in a recently operated pelvis.

Uterine artery embolization is an emerging technique which has become an effective and safe treatment for postpartum hemorrhage, allowing the preservation of reproductive function. Ultrasound guided injection of thrombin directly into the pseudoaneurysm has been used as a substitute for arterial embolization; however, its indications and effectiveness have not yet been determined [14]. The surgical approach may be more suitable in cases of acute and massive bleeding in which there is no time for embolization and may depend on the specific resources available.

\section{Conclusion}

Uterine artery pseudoaneurysm after hysterectomy is a very rare, but dangerous scenario. It usually presents with internal pelvic hemorrhage and can cause hemodynamic shock. The diagnosis can be confirmed either with pelvic angiography (CTA, MRA or DSA). A uterine artery or internal artery embolization is the preferred, and less invasive than surgical ligation.

Contributors: AS carried out the embolization procedure, wrote the endovascular section of manuscript, general arrangement of manuscript; MJ supervised and wrote the clinical aspects of the case. AS will act as a study guarantor. Both authors approved the final version of this manuscript.

Funding: None; Competing interests: None stated. 


\section{References}

1. Saad N, Saad W, Davies M, Waldman D, Fultz P, Rubens D. Pseudoaneurysms and the role of minimally invasive techniques in their management. Radiographics. 2005;25(Suppl 1):S173-S189.

2. Ho SP, Ong CL, Tan BS. A case of uterine artery pseudoaneurysms. Singapore Med J. 2002;43:202-204.

3. Nanjundan P, Rohilla M, Raveendran A, Jain V, Khandelwal N. Pseudoaneurysm of uterine artery: a rare cause of secondary postpartum hemorrhage, managed with uterine artery embolisation. J Clinical Imaging Science. 2011;1:1-4.

4. Takeda A, Kato K, Mori M, Sakai K, Mitsui T, Nakamura $\mathrm{H}$. Late massive uterine hemorrhage caused by ruptured uterine artery pseudoaneurysm after laparoscopicassisted myomectomy. J Minim Invasive Gynecol. 2008;15:212-216.

5. Asai S, Asada H, Furuya M, Ishimoto H, Tanaka M, Yoshimura Y. Pseudoaneurysm of the uterine artery after laparoscopic myomectomy. Fertil Steril. 2009;91:929. e1-3.

6. Lee WK, Roche CJ, Duddalwar VA, Buckley AR, Morris DC. Pseudoaneurysm of the uterine artery after abdominal hysterectomy: radiologic diagnosis and management. Am J Obstet Gynecol. 2001;185:12691272.

7. Higon MA, Domingo S, Bauset C, Martinez J, Pellicer A. Hemorrhage after myomectomy resulting from pseudoaneurysm of the uterine artery. Fertil Steril. 2007;87:417.e5-8.

8. Langer J, Cope C. Ultrasonographic diagnosis of uterine artery pseudoaneurysm after hysterectomy. J Ultrasound Med. 1999;18:711-714.

9. Kim YA, Han YH, Jun KC, Jeon MK, Lee ES. Uterine artery pseudoaneurysm manifesting delayed post abortal bleeding. Fertil Steril. 2008;90:849:e11-14.

10. Butori N, Coulange L, Filipuzzi L, Krausé D, Loffroy R. Pseudoaneurysm of the uterine artery after cesarean delivery: Management with superselective arterial embolization. Obstet Gynecol. 2009;113:540-543.

11. Polat P, Suma S, Kantarcý M, Alper F, Levent A. Color Doppler US in the evaluation of uterine vascular abnormalities. Radiographics. 2002;22:47-53.

12. Munera F, Soto JA, Palacio D, Velez SM, Medina E. Diagnosis of arterial injuries caused by penetrating trauma to the neck: comparison of helical CT angiography and conventional angiography. Radiology. 2000;216:356-362.

13. Vedantham S, Goodwin SC, McLucas B, Mohr G. Uterine artery embolization: An underused method of controlling pelvic hemorrhage. Am J Obstet Gynecol. 1997; 176:938-948.

14. Kovo M, Behar DJ, Friedman V, Malinger G. Pelvic arterial pseudoaneurysm-a rare complication of Cesarean section: Diagnosis and novel treatment. Ultrasound Obstet Gynecol. 2007;30:783-785. 